\title{
Some Variations on the Notion of Locally Testable Language
}

\author{
José Carlos Costa
}

July 2,1999

\begin{abstract}
The aim of this paper is to complete the characterization of the languages that are Boolean combinations (of a subset) of languages of the form $w A^{*}, A^{*} w$ or $L(w, r, t, n)$, where $A$ is an alphabet, $w \in A^{+}$, $r, t \geq 0, n \geq 1$ and $L(w, r, t, n)$ denotes the set of all words $u$ in $A^{+}$ such that the number of occurrences of the factor $w$ in $u$ is congruent to $r$ threshold $t \bmod n$. For each class $\mathcal{C}$ of languages such that $A^{+} \mathcal{C}$ is a Boolean algebra generated by some of the following types of languages: $w A^{*}, A^{*} w, A^{*} w A^{*}=L(w, 1,1,1)$ or $L(w, r, t, 1)$, and such that $\mathcal{C}$ does not constitute a variety of languages, we compute the smallest variety of languages containing $\mathcal{C}$ and the largest variety of languages contained in $\mathcal{C}$.
\end{abstract}

\section{Introduction}

In this paper we are interested in classes of languages $\mathcal{C}$ such that, for each alphabet $A$, the Boolean algebra $A^{+} \mathcal{C}$ is generated by some of the following types of languages: $w A^{*}, A^{*} w, A^{*} w A^{*}(=L(w, 1,1,1)), L(w, r, t, 1)$ or $L(w, r, t, n)$, where $w \in A^{+}, r, t \geq 0$ and $n \geq 1$. As an example we have the well-known class of locally testable languages, denoted $\mathcal{L} t$, which is such that $A^{+} \mathcal{L} t$ is the Boolean algebra generated by the languages of the form $w A^{*}, A^{*} w$ and $A^{*} w A^{*}$, where $w \in A^{+}$. The locally testable languages were characterized independently by Brzozowski and Simon [3] and McNaughton [6] as being those languages whose syntactic semigroup lies in LSl, the pseudovariety of all locally idempotent and locally commutative semigroups. Recall also that a language $L$ is locally testable if one can decide the membership of a given word $u$ in $L$ by considering the factors of a fixed length $k$ of $u$ and its prefixes and suffixes of length $<k$.

J. C. Costa CMAT, Dep. Matemática e Aplicações, Universidade do Minho, Campus de Gualtar, 4700-320 Braga, Portugal; e-mail: jcosta@math.uminho.pt 
In [2], Beauquier and Pin considered three variations on this last definition of locally testable languages and obtained three different classes of languages. First, they dropped the conditions about the prefixes and the suffixes and defined strongly locally testable $(\mathcal{S} l t)$ languages to be those languages whose elements are determined by factors of a fixed length. The class of all such languages in $A^{+}$is the Boolean algebra generated by the languages of the form $A^{*} w A^{*}$ with $w \in A^{+}$. This class is not a variety of languages but it is decidable and characterized by a nice algebraic property. In this paper we consider a class of languages intermediate between locally testable languages and strongly locally testable languages, which we call locally testable by prefixes $(\mathcal{L} t-p)$. Membership of a word $u$ in this type of language is determined by the factors of $u$ of a fixed length $k$ and by the prefixes of $u$ of length $<k$. Thus a language in $A^{+}$is locally testable by prefixes if it is a Boolean combination of languages of the form $w A^{*}$ and $A^{*} w A^{*}$ where $w \in A^{+}$. This class of languages is characterized by an algebraic property similar to that of Beauquier and Pin.

Secondly, Beauquier and Pin characterized the languages in $A^{+}$that are Boolean combinations of languages of the form $w A^{*}, A^{*} w$ or $L(w, r, t, 1)$, which they called threshold locally testable $(\mathcal{T} l t)$. Membership of a word $u$ in such a language is determined by the factors of $u$ of a fixed length $k$, but taking in account their number of occurrences up to a certain "threshold", and by the prefixes and suffixes of $u$ of length $<k$.

Finally, by dropping the conditions about the prefixes and the suffixes on this last condition, Beauquier and Pin introduced another class of languages whose elements, called strongly threshold locally testable $(\mathcal{S} t l t)$ languages, are Boolean combinations of languages of the form $L(w, r, t, 1)$. However, the syntactic characterization of these languages only recently was obtained by Pin [8]. Once again we describe a "lateralized" version of this work, by dropping only the condition about the suffixes. One obtains a class of languages whose elements are Boolean combinations of languages of the form $w A^{*}$ or $L(w, r, t, 1)$, which we call threshold locally testable by prefixes $(\mathcal{T} l t-p)$ languages.

If one replaces $w A^{*}$ by $A^{*} w$ on the generators of the languages "by prefixes" above, one obtains dually the classes of locally testable by suffixes $(\mathcal{L} t-s)$ and of threshold locally testable by suffixes ( $\mathcal{T} l t-s)$ languages. We complete our study by considering the languages that are Boolean combinations of languages of the form $w A^{*}, A^{*} w$ or $L(w, r, t, n)$. These languages, which we call counting locally testable $(\mathcal{C l t})$, were also characterized in [2]. Here, we show that they can also be obtained using only Boolean combinations of languages of the form $L(w, r, t, n)$, i.e., they coincide with its "strongly" version.

Now, we recall that the class $\mathcal{C}$ of all languages such that $A^{+} \mathcal{C}$ is the Boolean algebra generated by the set $\left\{w A^{*}: w \in A^{+}\right\}$(resp. $\left\{A^{*} w: w \in\right.$ $\left.A^{+}\right\},\left\{w A^{*}, A^{*} w: w \in A^{+}\right\}$) is already characterized (see [7], for instance). 
It is the class of languages associated, via Eilenberg's Theorem, with the pseudovariety $\mathbf{K}$ (resp. D, LI), consisting of all finite semigroups $S$ such that $e S=e$ (resp. $S e=e, e S e=e$ ) for each idempotent $e$ of $S$. This means that the characterization of the languages that are Boolean combinations (of a subset) of languages of the form $w A^{*}, A^{*} w, A^{*} w A^{*}$ or $L(w, r, t, n)$ is now complete.

In the last part of this paper we compute the smallest (resp. largest) variety of languages containing (resp. contained in) the classes of languages mentioned above and that are not varieties of languages. For instance, we show that the class of all $\mathcal{L} t$ (resp. $\mathcal{L} t$ and $\mathcal{J}$-trivial) languages is the smallest (resp. largest) variety of languages containing (resp. contained in) the class of all $\mathcal{S} l t$ languages. In other words, $\mathcal{L} t$ is generated (as a variety of languages) by the languages of the form $A^{*} w A^{*}$ with $w \in A^{+}$. We remark the analogy of this result with the well known characterization of the variety of languages $\mathcal{S} l$, associated with the pseudovariety $\mathbf{S l}$ of semilattices, as being the Boolean algebra generated by the languages of the form $A^{*} a A^{*}$ with $a \in A$.

\section{Preliminaries}

We begin by presenting basic definitions and notation concerning words and finite semigroups. Next we recall the notion of pseudovariety of semigroups and define the pseudovarieties mentioned in this paper. We then present the main definitions about recognizable languages and their relations with pseudovarieties. For omitted proofs and missing definitions, the reader is referred to the book of Pin [7].

\section{$2.1 \quad$ Words}

Let $A$ be a finite alphabet. We denote by $A^{+}$the set of non-empty words over $A$ and by $A^{*}$ the set $A^{+} \cup\{1\}$. If $u=a_{1} \cdots a_{k}\left(a_{i} \in A\right)$ is a word, the number $k$ is said the length of $u$ and is denoted by $|u|$. For each word $u$ of length $\geq k$, we denote by $p_{k}(u)$ (resp. $s_{k}(u)$ ) the prefix (resp. suffix) of $u$ of length $k$. For each word $u$, we denote by $i_{k}(u)$ (resp. $\left.t_{k}(u)\right)$ the word $u$ if $|w|<k$, and $p_{k}(u)$ (resp. $s_{k}(u)$ ) otherwise. We will denote by $F_{k}(u)$ the set of all factors of length $k$ of $u$.

For each pair of words $w$ and $u$, we denote by $\left[\begin{array}{l}w \\ u\end{array}\right]$ the number of occurrences of the factor $u$ in $w$. For instance $\left[\begin{array}{c}a b a a b a a a \\ a b a a\end{array}\right]=2$, since $a b a a$ occurs in two different places in abaabaaa: abaabaaa, abaabaaa.

Let us now introduce a congruence on the set of non-negative integers, which is crucial in what follows. Let $x, y, t \geq 0$ and $n \geq 1$ be integers. We say that, $x$ is congruent to $y$ threshold $t \bmod n$, denoted $x \equiv_{t, n} y$, if either $x=y$ or $x, y \geq t$ and $x$ is congruent to $y \bmod n$. For instance, the classes 
of $\equiv_{2,3}$ are $\{0\},\{1\},\{2,5,8, \ldots\},\{3,6,9, \ldots\}$ and $\{4,7,10, \ldots\}$.

For an alphabet $A$, a word $w \in A^{+}$, integers $r, t \geq 0$ and $n \geq 1$ set

$$
L(w, r, t, n)=\left\{u \in A^{+}:\left[\begin{array}{l}
u \\
w
\end{array}\right] \equiv_{t, n} r\right\} .
$$

For instance, $L(w, 1,1,1)=A^{*} w A^{*}$ and $L(a, 1,0,2)=B^{*} a B^{*}\left(a B^{*} a B^{*}\right)^{*}$, with $B=A \backslash\{a\}$, is the set of all words in $A^{+}$containing an odd number of occurrences of the letter $a \in A$.

\subsection{Pseudovarieties of semigroups}

Let $S$ be a finite semigroup and let $s \in S$. We denote by $s^{\omega}$ the unique idempotent of the subsemigroup of $S$ generated by $s$. We say that $S$ is aperiodic if $s^{\omega+1}=s^{\omega}$ for all $s \in S$.

Recall that a pseudovariety of semigroups is a class of finite semigroups closed under taking subsemigroups, homomorphic images and finite direct products. We denote by $\mathbf{A}$, Com, Acom, Sl and $\mathbf{J}$, respectively, the pseudovarieties of all finite aperiodic, commutative, aperiodic and commutative, idempotent and commutative (or semilattices) and $\mathcal{J}$-trivial semigroups. Particularly important in this paper is the pseudovariety LSI of all finite semigroups $S$ such that $e S e \in \mathbf{S l}$ for every idempotent $e \in S$.

It is well known, by Reiterman's Theorem [9], that every pseudovariety $\mathbf{V}$ is defined by a family $\Sigma$ of pseudoidentities, written $\mathbf{V}=\llbracket \Sigma \rrbracket$. We refer the reader to Almeida [1] for background on pseudovarieties and pseudoidentities. We have, for instance, the following equalities:

$$
\begin{array}{rlrl}
\mathbf{A} & =\llbracket x^{\omega+1}=x^{\omega} \rrbracket, & \mathbf{A c o m} & =\llbracket x^{\omega+1}=x^{\omega}, x y=y x \rrbracket \\
\mathbf{C o m} & =\llbracket x y=y x \rrbracket, & \mathbf{J}=\llbracket(x y)^{\omega}=(y x)^{\omega}, x^{\omega+1}=x^{\omega} \rrbracket \\
\mathbf{S} \mathbf{l} & =\llbracket x^{2}=x, x y=y x \rrbracket . & &
\end{array}
$$

Now, we recall three calculations of semidirect product of pseudovarieties of semigroups which will be used later. The first was obtained by Brzozowski and Simon [3] and McNaughton [6] and the two last ones by Thérien and Weiss [10].

$$
\begin{aligned}
\mathbf{S l} * \mathbf{D} & =\llbracket x^{\omega} y x^{\omega} y x^{\omega}=x^{\omega} y x^{\omega}, x^{\omega} y x^{\omega} z x^{\omega}=x^{\omega} z x^{\omega} y x^{\omega} \rrbracket=\mathbf{L S l} \\
\mathbf{C o m} * \mathbf{D} & =\llbracket x^{\omega} r y^{\omega} s x^{\omega} t y^{\omega}=x^{\omega} t y^{\omega} s x^{\omega} r y^{\omega} \rrbracket \\
\mathbf{A c o m} * \mathbf{D} & =(\mathbf{C o m} * \mathbf{D}) \cap \mathbf{A} .
\end{aligned}
$$

\subsection{Recognizable languages}

Let $A$ be an alphabet and let $\mathbf{V}$ be a pseudovariety. A subset $L$ of $A^{+}$is called a language. It is said to be recognizable (resp. $\mathbf{V}$-recognizable) if there 
exists a finite semigroup $S$ (resp. in $\mathbf{V}$ ) and a morphism $\mu: A^{+} \rightarrow S$ such that $L=\mu^{-1}(\mu(L))$. In that case, we say that $S$ recognizes $L$. The syntactic congruence of a language $L$ is the congruence $\sim_{L}$ over $A^{+}$given by

$u \sim_{L} v \quad$ if and only if $\quad x u y \in L \Leftrightarrow x v y \in L$ for all $x, y \in A^{*}$.

The syntactic semigroup of $L$, denoted by $S(L)$, is the quotient of $A^{+}$ by $\sim_{L}$. We know that $L$ is recognizable (resp. $\mathbf{V}$-recognizable) if and only if $S(L)$ is finite (resp. $S(L) \in \mathbf{V}$ ). The natural morphism $\eta: A^{+} \rightarrow S(L)$ is called the syntactic morphism of $L$ and $P=\eta(L)$ is its syntactic image. For more details on recognizable languages, the reader is referred to $[7,5]$.

A class of (recognizable) languages is a correspondence $\mathcal{C}$ associating with each alphabet $A$ a set $A^{+} \mathcal{C}$ of (recognizable) languages of $A^{+}$. A variety of languages is a class $\mathcal{V}$ of recognizable languages such that

(1) for every alphabet $A, A^{+} \mathcal{V}$ is closed under finite union, finite intersection and complement;

(2) for every morphism $\varphi: A^{+} \rightarrow B^{+}, L \in B^{+} \mathcal{V}$ implies $\varphi^{-1}(L) \in A^{+} \mathcal{V}$;

(3) if $L \in A^{+} \mathcal{V}$ and $a \in A$, then $a^{-1} L=\left\{u \in A^{+}: a u \in L\right\}$ and $L a^{-1}=\left\{u \in A^{+}: u a \in L\right\}$ are in $A^{+} \mathcal{V}$.

Let $\mathbf{V}$ be a pseudovariety and let $\mathcal{V}$ be the class of recognizable languages which associates with each alphabet $A$ the set $A^{+} \mathcal{V}$ of $\mathbf{V}$-recognizable languages of $A^{+}$. One can show that $\mathcal{V}$ is a variety of languages. Moreover, Eilenberg [5] proved the following fundamental result.

Theorem 2.1 The correspondence $\mathbf{V} \mapsto \mathcal{V}$ defines a bijective correspondence between pseudovarieties of semigroups and varieties of languages.

\section{Languages defined by factors of words}

In this section, we begin by presenting some equivalence relations which will be used to describe the languages we are interested in. We then present the characterizations of the languages.

\subsection{Some equivalence relations}

Let $k, n \geq 1$ and $t \geq 0$ be integers. We define an equivalence $\equiv_{k, t, n}$ of finite index on $A^{+}$by setting

$u \equiv_{k, t, n} v$ if and only if, for every word $x$ of length $\leq k,\left[\begin{array}{l}u \\ x\end{array}\right] \equiv_{t, n}\left[\begin{array}{l}v \\ x\end{array}\right]$.

For instance, if $u=a^{3} b a b a b a^{2}$ and $v=a^{2} b a b a b a b a^{3}$, we have $u \equiv_{3,2,1} v$ but $u \not \equiv_{3,2,2} v$ since $\left[\begin{array}{c}u \\ a b a\end{array}\right]=3 \not_{2,2} 4=\left[\begin{array}{c}v \\ a b a\end{array}\right]$. However $u \equiv_{3,2,2} a^{2} b a b a b a b a b a^{3}$. We note that $\equiv_{k, t, n}$ is not a congruence in general. For instance, consider 
$A=\{a, b\}, u=a b a$ and $v=a b a b$. One has $u \equiv_{2,1,1} v$, but $u a \equiv_{2,1,1} v a$. Indeed $a^{2}$ is a factor of length 2 of $u a$ but it is not a factor of $v a$.

Let now $\sim_{k, t, n}$ be the congruence of finite index on $A^{+}$given by $u \sim_{k, t, n} v$ if and only if $i_{k-1}(u)=i_{k-1}(v), t_{k-1}(u)=t_{k-1}(v)$ and $u \equiv_{k, t, n} v$.

If on the definition of $\sim_{k, t, n}$ we drop the condition about the suffixes we obtain a new equivalence on $A^{+}$, which we denote by $\approx_{k, t, n}$. That is, $\approx_{k, t, n}$ is given by

$$
u \approx_{k, t, n} v \text { if and only if } i_{k-1}(u)=i_{k-1}(v) \text { and } u \equiv_{k, t, n} v .
$$

This equivalence is not a congruence in general.

We say that an equivalence relation $\equiv$ on $A^{+}$saturates a language $L \subseteq$ $A^{+}$if $L$ is a union of $\equiv$-classes.

Definition 3.1 Let $A$ be an alphabet. We say that a language of $A^{+}$is

- locally testable (resp. strongly locally testable, locally testable by prefixes) if it is saturated by $\sim_{k, 1,1}$ (resp. $\equiv_{k, 1,1}, \approx_{k, 1,1}$ ) for some $k$;

- threshold locally testable (resp. strongly threshold locally testable, threshold locally testable by prefixes) if it is saturated by $\sim_{k, t, 1}$ (resp. $\equiv_{k, t, 1}, \approx_{k, t, 1}$ ) for some $k$ and $t$;

- counting locally testable (resp. strongly counting locally testable, counting locally testable by prefixes) if it is saturated by $\sim_{k, t, n}$ (resp. $\equiv_{k, t, n}, \approx_{k, t, n}$ ) for some $k, n$ and $t$.

The notions of locally testable by suffixes, threshold locally testable by suffixes and counting locally testable by suffixes can be defined dually by dropping the condition about the prefixes, instead of the suffixes, on the definition of $\sim_{k, t, n}$. We will use, respectively, the notations $\mathcal{L} t, \mathcal{T} l t, \mathcal{C} l$, $\mathcal{S} l t, \mathcal{L} t-p$, etc, either for the classes of all locally testable, threshold locally testable, counting locally testable, strongly locally testable, locally testable by prefixes, etc, languages, or for the languages themselves.

The next proposition describes these classes as Boolean algebras. For a set of languages $\mathcal{L}$ we denote by $\mathcal{B}(\mathcal{L})$ the Boolean algebra generated by $\mathcal{L}$.

Proposition 3.2 Let $A$ be an alphabet. Then

$$
\begin{aligned}
A^{+} \mathcal{C} l t & =\mathcal{B}\left\{w A^{*}, A^{*} w, L(w, r, t, n) \mid w \in A^{+}, r, t \geq 0, n \geq 1\right\}, \\
A^{+} \mathcal{C} l t-p & =\mathcal{B}\left\{w A^{*}, L(w, r, t, n) \mid w \in A^{+}, r, t \geq 0, n \geq 1\right\}, \\
A^{+} \mathcal{C} l t-s & =\mathcal{B}\left\{A^{*} w, L(w, r, t, n) \mid w \in A^{+}, r, t \geq 0, n \geq 1\right\}, \\
A^{+} \mathcal{S} c l t & =\mathcal{B}\left\{L(w, r, t, n) \mid w \in A^{+}, r, t \geq 0, n \geq 1\right\} .
\end{aligned}
$$

Similar results are valid for the four classes of "locally testable" languages and the four classes of "threshold locally testable" languages. We only need to substitute $L(w, r, t, n)$ by $L(w, 1,1,1)$ and by $L(w, r, t, 1)$, respectively. 
The next result shows that the generators of the form $w A^{*}$ and $A^{*} w$ are superfluous for $\mathcal{C} l t$. That is, we can restrict the generators of $\mathcal{C l t}$ to the languages of the form $L(w, r, t, n)$.

Proposition 3.3 We have the equalities $\mathcal{C} l t=\mathcal{C} l t-p=\mathcal{C} l t-s=\mathcal{S} c l t$.

Proof. It is clear that it suffices to prove the inclusion $\mathcal{C} l t \subseteq \mathcal{S}$ clt. For that, we will show that for each alphabet $A$ and each $w \in A^{+}$, the languages $w A^{*}$ and $A^{*} w$ are Boolean combinations of languages of the form $L(u, r, t, n)$ with $u \in A^{+}, r, t \geq 0$ and $n \geq 1$. To be more precise, we show that $w A^{*}$ (for $A^{*} w$ is similar) is the (disjoint) union of all languages of the form

$$
L(w, \alpha, 1,2) \cap \bigcap_{a \in A} L\left(a w, \beta_{a}, 0,2\right)
$$

where $\alpha \in\{1,2\}, \beta_{a} \in\{0,1\}, \sum_{a \in A} \beta_{a}$ is even if $\alpha=1$ and it is odd if $\alpha=2$.

We begin by observing that a word $u \in A^{+}$lies in $w A^{*}$ if and only if

$$
\sum_{a \in A}\left[\begin{array}{c}
u \\
a w
\end{array}\right]=\left[\begin{array}{c}
u \\
w
\end{array}\right]-1
$$

Let $u \in w A^{*}$. Then, either $\left[\begin{array}{l}u \\ w\end{array}\right]$ is odd, or it is even and not null. In the first case $u \in L(w, 1,1,2)$. Furthermore, we deduce from (1) that $\sum_{a \in A}\left[\begin{array}{c}u \\ a w\end{array}\right]$ is even. This implies that

$$
u \in \bigcap_{a \in A} L\left(a w, \beta_{a}, 0,2\right)
$$

for some family $\left(\beta_{a}\right)_{a \in A}, \beta_{a} \in\{0,1\}$, such that $\sum_{a \in A} \beta_{a}$ is even. Analogously, one can show that in the second case

$$
u \in L(w, 2,1,2) \cap \bigcap_{a \in A} L\left(a w, \beta_{a}, 0,2\right)
$$

for some family $\left(\beta_{a}\right)_{a \in A}, \beta_{a} \in\{0,1\}$, such that $\sum_{a \in A} \beta_{a}$ is odd. This shows one of the inclusions.

Conversely, if $u \in L(w, \alpha, 1,2) \cap \bigcap_{a \in A} L\left(a w, \beta_{a}, 0,2\right)$ where $\alpha$ and $\beta_{a}$ are in the conditions of the statement, we have that $\alpha$ (and so also $\left[\begin{array}{l}u \\ w\end{array}\right]$ ) is even if and only if $\sum_{a \in A} \beta_{a}$ is odd, that is, if and only if $\sum_{a \in A}\left[\begin{array}{c}u \\ a w\end{array}\right]$ is odd. Consequently $\left[\begin{array}{l}u \\ w\end{array}\right]$ and $\sum_{a \in A}\left[\begin{array}{l}u \\ a w\end{array}\right]$ are different whence $w$ is a prefix of $u$. So $u$ lies in $w A^{*}$.

We shall see that the other classes of languages are all distinct between themselves and from $\mathcal{C} l t$. The inclusion relation between them is as shown in the next figure. 


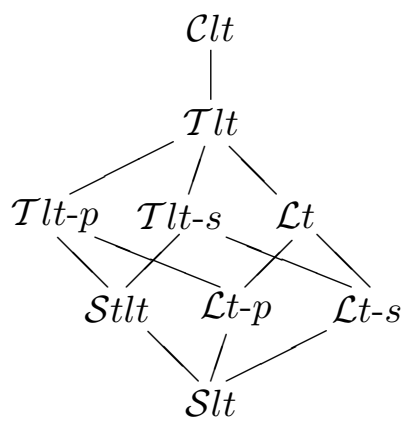

We shall also see that from these classes only $\mathcal{L} t, \mathcal{T} l t$ and $\mathcal{C} l t$ constitute varieties of languages. Furthermore we will prove that $\mathcal{L} t-p \cap \mathcal{L} t-s=\mathcal{S} l t$, that $\mathcal{T} l t-p \cap \mathcal{T} l t-s \neq \mathcal{S} t l t$ and that $\mathcal{L} t$ (resp. $\mathcal{T} l t)$ is the smallest variety of languages containing $\mathcal{S} l t$ (resp. Stlt).

Example 3.4 Let $A=\{a, b\}$. The language $L=b a^{*} b a^{*}$ is threshold locally testable by prefixes since

$$
L=b A^{*} \cap L(b, 2,3,1) .
$$

Less obvious is that $L$ is also strongly threshold locally testable. Indeed,

$$
L=L(b, 2,3,1) \backslash[L(a b, 2,2,1) \cup L(a b b, 1,1,1)]
$$

\subsection{Syntactic characterizations}

In this section we describe effective characterizations of the classes of languages presented in the previous section. These characterizations are all given in terms of an algebraic property of the syntactic morphisms of the languages. The classes $\mathcal{L} t, \mathcal{T} l t$ and $\mathcal{C} l t$ are characterized by a property of the syntactic semigroups of their languages. For the other classes it is also necessary to consider the syntactic images of the languages. This means by Eilenberg's Theorem that the first three classes are varieties of languages while the others are not.

We begin by presenting the characterizations of $\mathcal{L} t, \mathcal{T} l t$ and $\mathcal{C} l t$. The first is due to Brzozowski and Simon [3] and McNaughton [6]. The others are due to Beauquier and Pin [2].

Theorem 3.5 Let $L$ be a recognizable language.

(1) $L$ is $\mathcal{L} t$ if and only if $S(L) \in \mathbf{S} \mathbf{l} * \mathbf{D}$.

(2) $L$ is $\mathcal{T}$ lt if and only if $S(L) \in \mathbf{A c o m} * \mathbf{D}$.

(3) $L$ is $\mathcal{C} l t$ if and only if $S(L) \in \mathbf{C o m} * \mathbf{D}$. 
Thus, it is decidable whether a given language is $\mathcal{L} t, \mathcal{T} l t$ or $\mathcal{C l t}$.

We now proceed to describe characterizations of the remaining classes of languages. Let $S$ be a finite semigroup. Define $\equiv$ to be the smallest equivalence relation on $S$ containing the relation $\mathcal{J}$ and satisfying the condition:

$$
\forall e=e^{2}, f=f^{2} \in S \forall r, s \in S, \text { erfse } \equiv f \operatorname{serf} .
$$

Beauquier and Pin [2] and Pin [8] gave, respectively, the characterizations of the classes $\mathcal{S} l t$ and $\mathcal{S} t l t$.

Theorem 3.6 Let $L$ be a recognizable language of $A^{+}$, let $S$ be the syntactic morphism of $L$ and let $P$ be its syntactic image.

(1) $L$ is $\mathcal{S}$ lt if and only if $S \in \mathbf{L S l}$ and $P$ is a union of $\mathcal{J}$-classes of $S$.

(2) $L$ is Stlt if and only if $S \in \mathbf{A c o m} * \mathbf{D}$ and $P$ is a union of $\equiv$-classes of $S$.

We now present a "lateralized" version of this last theorem.

Theorem 3.7 Let $L$ be a recognizable language of $A^{+}$, let $S$ be the syntactic morphism of $L$ and let $P$ be its syntactic image.

(1) $L$ is $\mathcal{L} t-p$ if and only if $S \in \mathbf{L S l}$ and $P$ is a union of $\mathcal{R}$-classes of $S$.

$\left(1^{\prime}\right) L$ is $\mathcal{L} t$-s if and only if $S \in \mathbf{L S l}$ and $P$ is a union of $\mathcal{L}$-classes of $S$.

(2) $L$ is $\mathcal{T}$ lt-p if and only if $S \in \mathbf{A c o m} * \mathbf{D}$ and $P$ is a union of $\mathcal{R}$-classes of $S$.

$\left(2^{\prime}\right) L$ is $\mathcal{T}$ lt-s if and only if $S \in \mathbf{A c o m} * \mathbf{D}$ and $P$ is a union of $\mathcal{L}$-classes of $S$.

Proof. The proofs are adapted without difficulty from the corresponding proofs of Theorem 3.6. We only recall the proof of (2). Suppose first that $L$ is a $\mathcal{T} l t-p$ language. Then, $L$ is saturated by $\approx_{k, t, 1}$ for some $k$ and $t$. Since $\mathcal{T} l t-p \subseteq \mathcal{T} l t, L$ is also $\mathcal{T} l t$ and Theorem 3.5 shows that $S(L) \in \mathbf{A c o m} * \mathbf{D}$. Since the syntactic morphism $\eta: A^{+} \rightarrow S$ is onto, one can fix, for each element $s \in S^{1}$ a word $\bar{s} \in A^{*}$ such that $\eta(\bar{s})=s$ (if $s=1$, we take $\bar{s}=1$ ). To prove that $P$ is a union of $\mathcal{R}$-classes of $S$, let us consider two $\mathcal{R}$ equivalent elements $r$ and $s$ of $S$ and suppose that $r \in P$. We want to show that $s \in P$. Since $r \mathcal{R} s$ there exist $x, y \in S^{1}$ such that $r x=s$ and $s y=r$. Now, since $S$ is finite, there exists an integer $n$ such that, for any $s \in S, s^{n}$ is idempotent. Choosing one such $n \geq k t$, we have $\bar{r}(\bar{x} \bar{y})^{n} \approx_{k, t, 1} \bar{r}(\bar{x} \bar{y})^{n} \bar{x}$. But $\eta\left(\bar{r}(\bar{x} \bar{y})^{n}\right)=r \in P$ and thus $\bar{r}(\bar{x} \bar{y})^{n} \in L$. This implies $\bar{r}(\bar{x} \bar{y})^{n} \bar{x} \in L$, whence $\eta\left(\bar{r}(\bar{x} \bar{y})^{n} \bar{x}\right)=s \in P$.

Conversely, since $S \in \mathbf{A c o m} * \mathbf{D}$ it follows from Theorem 3.5 that $L$ is saturated by $\sim_{k, t, 1}$ for some $k$ and $t$. We will show that $L$ is saturated 
by $\approx_{k, T, 1}$ for some $T$ sufficiently large (more precisely, one can take $T \geq$ $\left.\left(1+t \cdot\left(|A|^{k}\right) !\right)(1+|A|)\right)$. To each word $w$ we associate a labeled graph $N(w)$ where the set of vertices is $F_{k-1}(w)$ and if $u \in F_{k}(w)$, there exists an edge of label $\left[\begin{array}{c}w \\ u\end{array}\right]$ threshold $t$ from $p_{k-1}(u)$ to $s_{k-1}(u)$. The vertex $p_{k-1}(w)$ (resp. $\left.s_{k-1}(w)\right)$ is called the initial (resp. final) vertex of $N(w)$.

Let $w$ and $w^{\prime}$ be two words such that $w \approx_{k, T, 1} w^{\prime}$ and $w \in L$. We want to show that $w^{\prime} \in L$. If $|w|<k$ (or $\left|w^{\prime}\right|<k$ ), then $w=w^{\prime}$. So, we may suppose $|w|,\left|w^{\prime}\right| \geq k$. Suppose now that $|w|<T$. We claim that $w \sim_{k, T, 1} w^{\prime}$. Since $w \approx_{k, T, 1} w^{\prime}$, it remains to prove that $s_{k-1}(w)=s_{k-1}\left(w^{\prime}\right)$. If $k=1$ this is clear. Consider now $k \geq 2$ and put $s=s_{k-1}(w)$. Since $|w|<T$ we have $\left[\begin{array}{c}w \\ s\end{array}\right]=\left[\begin{array}{c}w^{\prime} \\ s\end{array}\right]<T$. Put $n=\left[\begin{array}{c}w \\ s\end{array}\right]$ and suppose that $s_{k-1}\left(w^{\prime}\right) \neq s$. Then

$$
\sum_{a \in A}\left[\begin{array}{c}
w \\
s a
\end{array}\right]=n-1 \quad \text { and } \quad \sum_{a \in A}\left[\begin{array}{l}
w^{\prime} \\
s a
\end{array}\right]=n .
$$

But this contradicts the assumption that $w \approx_{k, T, 1} w^{\prime}$, since in this case $\left[\begin{array}{c}w \\ s a\end{array}\right]$ $=\left[\begin{array}{c}w^{\prime} \\ s a\end{array}\right]$ for all $a \in A$. Then $s_{k-1}(w)=s_{k-1}\left(w^{\prime}\right)$ and the claim is proved. It follows that $w \sim_{k, t, 1} w^{\prime}$, since $t<T$, and we may conclude that $w^{\prime} \in L$.

Thus, we may assume that $|w|,\left|w^{\prime}\right| \geq T$. Since $\left[\begin{array}{c}w \\ u\end{array}\right] \equiv_{t, 1}\left[\begin{array}{c}w^{\prime} \\ u\end{array}\right]$ for any word $u$ of length $k$ and since $p_{k-1}(w)=p_{k-1}\left(w^{\prime}\right)$, the labeled graphs $N(w)$ and $N\left(w^{\prime}\right)$ are equal, except possibly for the final vertices. We denote by $f$ and $f^{\prime}$, respectively, the final vertices of $N(w)$ and $N\left(w^{\prime}\right)$.

We say that two vertices $v_{1}$ and $v_{2}$ are in the same strongly $t$-component, if there are two oriented paths from $v_{1}$ to $v_{2}$ and from $v_{2}$ to $v_{1}$ using only edges of label $t$. Since $N(w)$ and $N\left(w^{\prime}\right)$ have the same initial vertex, one has (see the proof of $\left[8\right.$, Theorem 3.3]) that $f$ and $f^{\prime}$ are in the same $t$ component. Now, one can show that $\eta(w) \mathcal{R} \eta\left(w^{\prime}\right)$. Since $P$ is union of $\mathcal{R}$-classes, we deduce that $\eta\left(w^{\prime}\right) \in P$ and thus that $w^{\prime} \in L$, which concludes the proof.

Since each $\mathcal{J}$-class of a finite semigroup is a union of $\mathcal{R}$-classes and a union of $\mathcal{L}$-classes, we have the following consequences of the last theorem.

Corollary 3.8 Let $L$ be a recognizable language of $A^{+}$, let $S$ be the syntactic morphism of $L$ and let $P$ be its syntactic image.

(1) $L$ is both $\mathcal{L} t-p$ and $\mathcal{L} t-s$ if and only if $L$ is $\mathcal{S} l$.

(2) $L$ is both $\mathcal{T}$ lt-p and $\mathcal{T}$ lt-s if and only if $S \in \mathbf{A c o m} * \mathbf{D}$ and $P$ is a union of $\mathcal{J}$-classes of $S$.

We remark that a language $L$ being both $\mathcal{T} l t-p$ and $\mathcal{T} l t-s$ does not imply that $L$ is $\mathcal{S} t l t$, that is, the class $\mathcal{T} l t-p \cap \mathcal{T} l t-s$ strictly contains the class $\mathcal{S} t l t$, as it is shown in the next example.

Example 3.9 Let $A=\{a, b\}$ and let $L=a^{*} b^{+} a^{*}$. Then $L$ is recognized by the following automaton. 


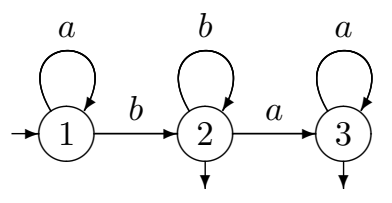

The syntactic semigroup of $L$ is defined by the relations $a^{2}=a, b^{2}=b$ and $b a b=0$. Its $\mathcal{J}$-class structure is represented in the following diagram, where the grey boxes represent the syntactic image $P$ of $L$.
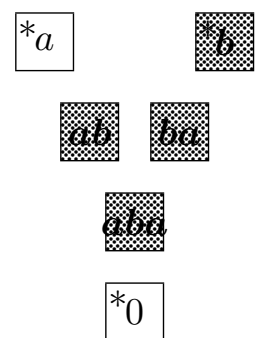

Thus $P$ is a union of $\mathcal{J}$-classes of $S(L)$ and $L$ is $\mathcal{T} l t-p$ and $\mathcal{T} l t$-s. Indeed, we have

$$
\begin{aligned}
L & =b^{+} a^{*} \cup a^{+} b^{+} a^{*}=\left[b A^{*} \backslash L(a b, 1,1,1)\right] \cup\left[a A^{*} \cap L(a b, 1,1,2)\right] \\
& =a^{*} b^{+} \cup a^{*} b^{+} a^{+}=\left[A^{*} b \backslash L(b a, 1,1,1)\right] \cup\left[A^{*} a \cap L(b a, 1,1,2)\right] .
\end{aligned}
$$

Let us now verify that $L$ is not $\mathcal{S}$ tlt. We prove that $P$ is not a union of $\equiv$-classes. Indeed, by definition of $\equiv$ and since $a$ and $b$ are idempotents, we have aabaa $\equiv$ baaab, that is, aba $\equiv 0$. Thus $P$ is not a union of $\equiv$-classes since $a b a \in P$ and $0 \notin P$.

Note also that $L$ is not $\mathcal{L} t$, since $S(L) \notin \mathbf{L S l}$. Indeed, for instance, a is idempotent and aba is not.

Example 3.10 Let $A=\{a, b, c\}$, and let $L=(a b)^{+} \cup a(b a)^{*} \cup\left\{c^{2}\right\}$. Then, $L$ is recognized by the following automaton.

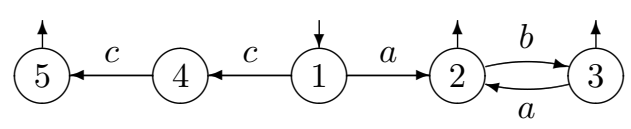

The syntactic semigroup $S(L)$ has seven elements and it is defined by the relations $a^{2}=a c=b^{2}=b c=c a=c b=c^{3}=0$. Its $\mathcal{J}$-class structure is represented in the following figure.

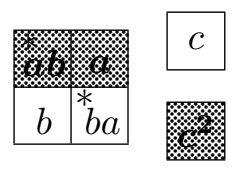


As one can show, $S(L) \in \mathbf{L S}$. On the other hand, the syntactic image of $L$ is the set $P=\left\{a b, a, c^{2}\right\}$. Since it is a union of $\mathcal{R}$-classes of $S(L), L$ is $\mathcal{L} t-p$. Indeed, we can write

$$
L=\left\{a, c^{2}\right\} A^{*} \backslash A^{*}\left\{a^{2}, a c, b^{2}, b c, c a, c b, c^{3}\right\} A^{*} .
$$

Note that $P$ is not a union of $\mathcal{L}$-classes of $S(L)$. So $L$ is not $\mathcal{L} t$-s.

\section{The varieties of languages generated}

In this section we compute the smallest (resp. largest) variety of languages containing (resp. contained in) each one of the classes of languages considered in the last section.

As we have seen in Theorem 3.5, the classes $\mathcal{L} t$ of locally testable languages and $\mathcal{T} l t$ of threshold locally testable languages are varieties of languages. Let us prove the following result.

Proposition 4.1 The class $\mathcal{L} t$ (resp. $\mathcal{T} l$ t) is the smallest variety of languages containing the languages of the form $A^{*} w A^{*}$ (resp. $L(w, r, t, 1)$ ) for any alphabet $A$ and $w \in A^{+}$(resp. and $r, t \geq 0$ ).

Proof. We only give the proof for $\mathcal{L} t$. The proof for $\mathcal{T} l t$ is a consequence of this one since $A^{*} w A^{*}=L(w, 1,1,1)$. Let $\mathcal{V}$ be the smallest variety of languages containing the languages of the form $A^{*} w A^{*}$, where $A$ is any alphabet and $w \in A^{+}$. First, it is clear that $\mathcal{V}$ is contained in $\mathcal{L} t$ since for every alphabet $A$ and $w \in A^{+}$, the language $A^{*} w A^{*}$ is locally testable.

Let now $A$ be a fixed alphabet and let $L \in A^{+} \mathcal{L} t$. Then $L$ is a Boolean combination of languages of the form $w A^{*}, A^{*} w A^{*}$ or $A^{*} w$, where $w \in A^{+}$. Thus, to prove that $L \in A^{+} \mathcal{V}$ it suffices to show that each one of these languages lies in $A^{+} \mathcal{V}$. This is clear for every language of the form $A^{*} w A^{*}$, by definition of $\mathcal{V}$. Consider now a language of the form $w A^{*}$. Let $B$ be the alphabet obtained from $A$ by the addition of a new letter $b$, i.e., $B=A \cup\{b\}$. The language $B^{*} b w B^{*}$ lies in $B^{+} \mathcal{V}$. Then the language

$$
b^{-1}\left(B^{*} b w B^{*}\right)=B^{*} b w B^{*} \cup w B^{*}
$$

is also in $B^{+} \mathcal{V}$, since $B^{+} \mathcal{V}$ is closed under cancellation. Now,

$$
\left(B^{*} b w B^{*} \cup w B^{*}\right) \backslash B^{*} b B^{*}=w A^{*}
$$

is also a language of $B^{+} \mathcal{V}$, since $B^{*} b B^{*} \in B^{+} \mathcal{V}$ and $B^{+} \mathcal{V}$ is closed under complementation. Consider now the morphism $\varphi: A^{+} \rightarrow B^{+}$given by $\varphi(a)=a$ for all $a \in A$. We have $\varphi^{-1}\left(w A^{*}\right)=w A^{*}$, whence $w A^{*}$ is a language of $A^{+} \mathcal{V}$, since $\mathcal{V}$ is closed under inverse image of morphisms between free semigroups. That every language of the form $A^{*} w$, with $w \in A^{+}$, lies in $A^{+} \mathcal{V}$ can be proved analogously. So, we deduce that $L \in A^{+} \mathcal{V}$ and, 
consequently, that $A^{+} \mathcal{L} t \subseteq A^{+} \mathcal{V}$. Since this holds for all alphabets $A$, we conclude that $\mathcal{L} t \subseteq \mathcal{V}$.

Corollary 4.2 The class $\mathcal{L} t$ (resp. $\mathcal{T}$ lt) is the variety of languages generated by each of the classes $\mathcal{S} l t, \mathcal{L} t-p$ and $\mathcal{L} t-s$ (resp. Stlt, $\mathcal{T} l t-p$ and $\mathcal{T} l t-s)$.

This result and Proposition 3.3 imply the following corollary.

Corollary 4.3 The pseudovariety LSl (resp. Acom $* \mathbf{D}, \quad \mathbf{C o m} * \mathbf{D})$ is generated by the syntactic semigroups of the languages of the form $A^{*} w A^{*}$ (resp. $L(w, r, t, 1), L(w, r, t, n))$, where $A$ is any alphabet and $w \in A^{+}$(resp. and $r, t \geq 0$ and $n \geq 1)$.

Now we consider varieties of languages contained in the classes of languages we are studying. Let us begin by considering the equivalence relation 三 defined immediately before Theorem 3.6 and show the following observation.

Lemma 4.4 Let $S$ be a finite semigroup. Then, $S$ is $\equiv$-trivial if and only if $S$ lies in the pseudovariety $\mathbf{W}=\mathbf{J} \cap \llbracket x^{\omega} z y^{\omega} t x^{\omega}=y^{\omega} t x^{\omega} z y^{\omega} \rrbracket$.

Proof. By definition of the equivalence $\equiv, S$ is $\equiv$-trivial if and only if $S$ is $\mathcal{J}$-trivial (since $\mathcal{J}$ is contained in $\equiv$ ) and, for all idempotents $e, f \in S$ and all $r, s \in S$, erfse $=f \operatorname{ser} f$. It follows that $S$ is $\equiv$-trivial if and only if $S \in \mathbf{J}$ and $S$ satisfies the pseudoidentity $x^{\omega} z y^{\omega} t x^{\omega}=y^{\omega} t x^{\omega} z y^{\omega}$, that is, if and only if $S \in \mathbf{W}$.

Now we can prove our last result.

Proposition 4.5 (1) The class $\mathcal{L} t \cap \mathcal{J}$ (resp. $\mathcal{L} t \cap \mathcal{R}, \mathcal{L} t \cap \mathcal{L})$ of $\mathcal{L} t$ and $\mathcal{J}$-trivial (resp. $\mathcal{L}$ t and $\mathcal{R}$-trivial, $\mathcal{L} t$ and $\mathcal{L}$-trivial) languages is the largest variety of languages contained in the class $\mathcal{S} l$ (resp. $\mathcal{L} t$-p, $\mathcal{L} t-s)$.

(2) The class $\mathcal{T} l t \cap \mathcal{W}$ (resp. $\mathcal{T} l t \cap \mathcal{R}, \mathcal{T} l t \cap \mathcal{L})$ of $\mathcal{T} l t$ and $\equiv$-trivial (resp. $\mathcal{T}$ lt and $\mathcal{R}$-trivial, $\mathcal{T}$ lt and $\mathcal{L}$-trivial) languages is the largest variety of languages contained in the class $\mathcal{S}$ tlt (resp. $\mathcal{T} l t-p, \mathcal{T} l t-s)$.

Proof. We only give the proof of (2) for $\mathcal{T} l t \cap \mathcal{W}$. The other cases are similar. Let $\mathcal{V}$ be the largest variety of languages contained in the class of all strongly threshold locally testable languages and let $L \in \mathcal{T} l t \cap \mathcal{W}$. Then $S(L) \in(\mathbf{A} \operatorname{com} * \mathbf{D}) \cap \mathbf{W}$. In particular, $S(L) \in \mathbf{A c o m} * \mathbf{D}$ and the syntactic image of $L$ is a union of $\equiv$-classes of $S(L)$, since they are trivial by Lemma 4.4. Hence, by Theorem 3.6, $L$ is strongly threshold locally testable. So, by definition of $\mathcal{V}$, we have $\mathcal{T} l t \cap \mathcal{W} \subseteq \mathcal{V}$.

Let now $A$ be an alphabet and let $L \in A^{+} \mathcal{V}$. Then $L$ is strongly threshold locally testable, whence $L$ is also threshold locally testable. Now, we prove 
that $L$ is $\equiv$-trivial. By Lemma 4.4, we have to show that $L$ is $\mathcal{J}$-trivial and that $S(L)$ satisfies the pseudoidentity $x^{\omega} z y^{\omega} t x^{\omega}=y^{\omega} t x^{\omega} z y^{\omega}$.

Suppose first, by way of contradiction, that $L$ is not $\mathcal{J}$-trivial, that is, suppose that $S(L)$ does not verify the pseudoidentity $(x y)^{\omega}=(y x)^{\omega}$. Then, there exist $u, v \in A^{+}$such that $(u v)^{n} \chi_{L}(v u)^{n}$ for all $n \geq 1$. Hence, for each $n \geq 1$, there exist $r_{n}, s_{n} \in A^{*}$ such that either $r_{n}(u v)^{n} s_{n} \in L$ and $r_{n}(v u)^{n} s_{n} \notin L$, or $r_{n}(u v)^{n} s_{n} \notin L$ and $r_{n}(v u)^{n} s_{n} \in L$. Then, either $(u v)^{n} \in$ $r_{n}^{-1} L s_{n}^{-1}$ and $(v u)^{n} \notin r_{n}^{-1} L s_{n}^{-1}$, or $(u v)^{n} \notin r_{n}^{-1} L s_{n}^{-1}$ and $(v u)^{n} \in r_{n}^{-1} L s_{n}^{-1}$. Let $k, t \geq 1$ and let $n \geq k t$. Then, we have $(u v)^{n} \equiv_{k, t, 1}(v u)^{n}$. So, for all $k, t \geq 1, r_{n}^{-1} L s_{n}^{-1}$ is not saturated by the equivalence $\equiv_{k, t, 1}$. This implies that $r_{n}^{-1} L s_{n}^{-1}$ is not strongly threshold locally testable. But this is absurd since $r_{n}^{-1} L s_{n}^{-1} \in A^{+} \mathcal{V}$ since $L \in A^{+} \mathcal{V}$ and $A^{+} \mathcal{V}$ is closed under cancellation. So $L$ must be $\mathcal{J}$-trivial.

Let us now show that $S(L)$ satisfies the pseudoidentity $x^{\omega} z y^{\omega} t x^{\omega}=$ $y^{\omega} t x^{\omega} z y^{\omega}$. Since $L$ is $\mathcal{S} t l t, S(L)$ is aperiodic by Theorem 3.5. So, there is an integer $m$ such that, for all $s \in S(L), s^{m}=s^{m+1}$. Suppose that $S(L)$ does not satisfy $x^{\omega} z y^{\omega} t x^{\omega}=y^{\omega} t x^{\omega} z y^{\omega}$, that is, suppose that there are $u, v, p, q \in A^{+}$such that $u^{n} p v^{n} q u^{n} \chi_{L} v^{n} q u^{n} p v^{n}$ for all $n \geq m$. Then, without loss of generality, we may suppose that there are $r_{n}, s_{n} \in A^{*}$ such that $r_{n} u^{n} p v^{n} q u^{n} s_{n} \in L$ and $r_{n} v^{n} q u^{n} p v^{n} s_{n} \notin L$. Hence, $u^{n} p v^{n} q u^{n} \in r_{n}^{-1} L s_{n}^{-1}$ and $v^{n} q u^{n} p v^{n} \notin r_{n}^{-1} L s_{n}^{-1}$. Let $k, t \geq 1$ and let $n \geq \max \{k t, m\}$. We have $u^{n} p v^{n} q u^{n} \equiv_{k, t, 1} v^{n} q u^{n} p v^{n}$ and, consequently, $r_{n}^{-1} L s_{n}^{-1}$ is not strongly threshold locally testable. But this is a contradiction by the same reasons as above and so $S(L)$ must satisfy the pseudoidentity $x^{\omega} z y^{\omega} t x^{\omega}=y^{\omega} t x^{\omega} z y^{\omega}$.

By Lemma 4.4 we deduce that $L$ is $\equiv$-trivial, which shows that $L \in$ $A^{+}(\mathcal{T} l t \cap \mathcal{W})$. We have proved that $A^{+} \mathcal{V} \subseteq A^{+}(\mathcal{T} l t \cap \mathcal{W})$ and since this holds for every alphabet $A$ we conclude that $\mathcal{V} \subseteq \mathcal{T} l t \cap \mathcal{W}$.

We summarize in the next diagram the inclusion relations stated in the results of this section. The emboldened classes are the varieties of languages and we denote by $\mathcal{T} l t-p s$ the class $\mathcal{T} l t-p \cap \mathcal{T} l t-s$. 


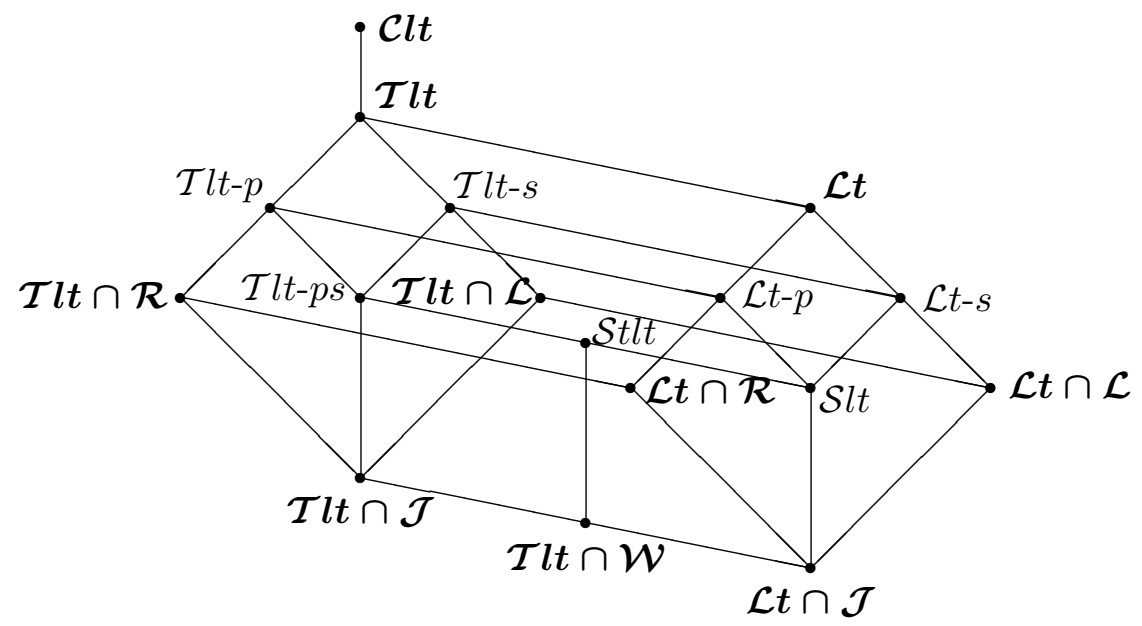

\section{References}

[1] J. Almeida, Finite Semigroups and Universal Algebra, World Scientific, Singapore, 1994.

[2] D. Beauquier and J.-E. Pin, Languages and scanners, Theoretical Computer Science 84 (1991) 3-21.

[3] J. Brzozowski and I. Simon, Characterization of locally testable events, Discrete Mathematics 4 (1973) 243-271.

[4] J. Costa, Quelques intersections de variétés de semigroupes finis et de variétés de langages, opérations implicites, Ph.D. Thesis, Université Paris 6, 1998.

[5] S. Eilenberg, Automata, languages and machines, vol. B, Academic Press, New York, 1976.

[6] R. McNaughton, Algebraic decision procedures for local testability, Math. Systems and Theory 8 (1974) 60-76.

[7] J.-E. Pin, Varieties of formal languages, Plenum, New York and North Oxford, London, 1986.

[8] J.-E. Pin, The expressive power of existential first order sentences of Büchi's sequential calculus, in Proc. $23^{\text {rd }}$ ICALP, Lecture Notes in Computer Science 1099, Springer, Berlin (1996) 300-311.

[9] J. Reiterman, The Birkhoff theorem for finite algebras, Algebra Universalis 14 (1982) 1-10.

[10] D. Thérien and A. Weiss, Graph congruences and wreath products, J. Pure Appl. Algebra 36 (1985) 205-215. 